\title{
PROJECTIONS TO AUTOMORPHIC FUNCTIONS ${ }^{1}$
}

\author{
C. J. EARLE AND A. MARDEN
}

1. The main results. Let $\bar{R}$ be a compact bordered Riemann surface with interior $R$. We represent $R$ as the orbit space $\Delta / G$ where $G$ is a finitely generated Fuchsian group of the second kind acting on the unit disk $\Delta$. Choose a fundamental polygon $R$ for $G$ in $\Delta$ whose closure $\bar{R}$ in the plane meets the boundary of $\Delta$ in a finite number of arcs, each of which corresponds to a boundary contour of $R$.

On the set of analytic functions in $\Delta$ we will consider the norms

$$
\begin{gathered}
\|f\|_{\infty}=\sup \{|f(z)|: z \in \Delta\}, \\
\|f\|=\iint_{\Delta}|f(z)| d x d y,
\end{gathered}
$$

and the corresponding Banach spaces

$$
H^{\infty}(\Delta)=\left\{f:\|f\|_{\infty}<\infty\right\} \text { and } A(\Delta)=\{f:\|f\|<\infty\} .
$$

We shall also consider the subspaces $H^{\infty}(G) \subset H^{\infty}(\Delta)$ and $A(G) \subset A(\Delta)$ of functions which satisfy

$$
f(A z)=f(z) \text { for all } A \in G \text { and } z \in \Delta .
$$

If $f$ satisfies (3), then

$$
\iint_{\Delta}|f(z)| d x d y=\iint_{\mathcal{R}}|f(z)|\left(\sum_{A \in G}\left|A^{\prime}(z)\right|^{2}\right) d x d y
$$

so that $A(G)$ consists of those analytic functions which satisfy (3) and are summable over $R$ with respect to the measure

$$
d m(z)=\sum_{A \in G}\left|A^{\prime}(z)\right|^{2} d x d y .
$$

Thus $H^{\infty}(G)$ corresponds to the space of bounded analytic functions on $R$, and $A(G)$ to the space of analytic functions on $R$ which are summable with respect to $d m$.

THEOREM. There is a projection $P$, bounded in the respective norms, which sends $A(\Delta)$ (resp. $H^{\infty}(\Delta)$ ) onto $A(G)$ (resp. $H^{\infty}(G)$ ) and has the following property: if $f(\zeta)=f(A \zeta)$ for some $\zeta \in \Delta$ and all $A \in G, f \in A(\Delta)$, then for any $g \in A(\Delta)$

Received by the editors December 15, 1966.

1 This research was supported by the National Science Foundation under grants GP 6145 and GP 3904. 


$$
(P f g)(\zeta)=f(\zeta) P g(\zeta)
$$

In particular, if $f \in A(G)$,

$$
P(f g)=f P g .
$$

This Theorem is a direct consequence of the following.

Lemma. There is a polynomial $F(z)$ such that the Poincaré series

$$
\Theta F(z)=\sum_{A \in G} F(A z) A^{\prime}(z)^{2}
$$

is bounded away from zero in the fundamental polygon $R$.

A somewhat less general form of the Theorem is due to Forelli [6] who obtained a bounded projection $P$ of $H^{\infty}(\Delta)$ onto $H^{\infty}(G)$ having property (7).

2. Proof of the Lemma. The set of limit points of $G$ is a closed subset of the unit circle of linear measure zero. If $\Omega$ is the complement of the set of limit points in the extended plane, then $\Omega / G$ is a compact Riemann surface, the double of $R$. Let $R^{*} \supset \bar{R}$ be a subsurface of $\Omega / G$ such that (i) $R^{*}$ is bounded by analytic curves, (ii) each component of $R^{*}-\bar{R}$ is a topological annulus, and (iii) $\pi(\infty)$ is in the exterior of $R^{*}$ where $\pi: \Omega \rightarrow \Omega / G$ is the natural map. Then $\pi^{-1}\left(R^{*}\right)=D^{*}$ contains $\Delta$, is invariant under $G$, and is bounded by a Jordan curve which is the union of $\pi^{-1}\left(\partial R^{*}\right)$ and the set of limit points of $G$. Moreover, $\bar{R}$ is a compact subset of $D^{*}$. Let $\Omega^{*}$ be a fundamental "polygon" for $G$ in $D^{*}$ ( $\Omega^{*}$ can be obtained, for example, by mapping $D^{*}$ on to $\Delta)$.

By Abel's theorem there exists a meromorphic differential $\omega$ on the compact surface $\Omega / G$ which is analytic and nonzero on the closure of $R^{*}$. The quadratic differential $\omega^{2}$ can be lifted to $D^{*}$ to determine an analytic $\phi(z)$ which is nonzero in $D^{*}$ and satisfies

$$
\phi(A z) A^{\prime}(z)^{2}=\phi(z) \quad \text { for all } z \in D^{*}, A \in G .
$$

Furthermore, since $\omega^{2}$ is analytic in the closure of $R^{*}$,

$$
\iint_{R^{*}}|\phi(z)| d x d y<\infty .
$$

We now appeal to a recent theorem of Bers [3] concerning Poincaré series in $D^{*}$. Let $Q(G)$ denote the Banach space of all functions $\phi(z)$ analytic in $D^{*}$ which satisfy (9) and (10), the norm being given by (10). Bers has proved [3, Theorem 2] that the Poincaré series (8) defines a continuous map of $A\left(D^{*}\right)$ onto $Q(G)$. (A short proof of this 
theorem can be found in [4].) Furthermore, since $D^{*}$ is a Jordan region, a theorem of $\mathrm{O}$. J. Farrell [5] implies that the polynomials are dense in $A\left(D^{*}\right)$.

Applying these results to our nonzero function $\phi(z)$ in $Q(G)$, we obtain a sequence $\left\{F_{n}\right\}$ of polynomials such that $\Theta F_{n} \rightarrow \phi$ in $Q(G)$. But convergence in $Q(G)$ implies uniform convergence on compact sets in $D^{*}$. In particular, $\Theta F_{n} \rightarrow \phi$ uniformly in $R$, and for sufficiently large $n, \Theta F_{n}$ is bounded away from zero in R. q.e.d.

3. Proof of the Theorem. Choose a polynomial $F(z)$ in accordance with the Lemma. For $f \in A(\Delta)$ define

$$
P f(z)=(\Theta F f)(z) / \Theta F(z) .
$$

Set

$$
\begin{aligned}
\delta^{-1} & =\inf \{|\Theta F(z)|: z \in \Omega\}>0, \\
M & =\sup \left\{\sum_{A \in G}\left|A^{\prime}(z)\right|^{2}: z \in \Omega\right\}<\infty .
\end{aligned}
$$

Then

$$
\begin{aligned}
\iint_{\mathcal{R}}|\Theta F f| d x d y & \leqq\|F\|_{\infty} \iint_{\Omega} \sum_{A \in G}|f(A z)|\left|A^{\prime}(z)\right|^{2} d x d y \\
& =\|F\|_{\infty} \sum_{A \in G} \iint_{A(\Omega)}|f(z)| d x d y=\|F\|_{\infty}\|f\| .
\end{aligned}
$$

Hence the series $\Theta F f$ converges absolutely and uniformly on compact subsets of $R$ and therefore on compact subsets of $\Delta$. Furthermore by the Lemma, $\Theta F$ is nonzero on $\Delta$. Consequently, $P f$ is analytic in $\Delta$; $P f$ obviously satisfies (3) and (6). We also have from (4) and (12) that

$$
\begin{aligned}
\|P f\| & =\iint_{\Delta}|P f(z)| d x d y=\iint_{Q}|P f(z)| d m(z) \\
& \leqq M \iint_{\mathcal{R}}|P f(z)| d x d y \leqq M \delta \iint_{\mathcal{R}}|\Theta F f| d x d y \\
& \leqq M \delta\|F\|_{\infty}\|f\|,
\end{aligned}
$$

and, if $f$ is in $H^{\infty}(\Delta)$ as well,

$$
\begin{aligned}
\|P f\|_{\infty} & =\sup \{|P f(z)|: z \in \Delta\} \\
& =\sup \{|P f(z)|: z \in \Omega\} \leqq M \delta\|F\|_{\infty}\|f\|_{\infty} .
\end{aligned}
$$

The remainder of the Theorem follows at once. q.e.d. 
4. Applications. As Forelli has pointed out [6, Corollary 2], Carleson's solution of the corona problem for $H^{\infty}(\Delta)$ and the existence of a projection $P: H^{\infty}(\Delta) \rightarrow H^{\infty}(G)$ with property (7) yield a solution of the corona problem for the compact bordered surface $R$. We state this consequence of Theorem 1 as

CoROLlary 1. Let $\operatorname{Mr}(R)$ be the maximal ideal space of the algebra $H^{\infty}(G)$. Then $R$ is dense in $\operatorname{Mr}(R)$.

Other proofs of Corollary 1 have been given in [1], [6], [7].

CoROllaRy 2. Let $S$ be a set of points in $\Delta$ which is invariant under $G$ and let $\zeta(z)$ be a complex valued function on $S$ such that $\zeta(A z)=\zeta(z)$ for all $A \in G$ and $z \in S$. There exists $f \in A(G)\left(\right.$ resp. $\left.H^{\infty}(G)\right)$ with $f(z)=\zeta(z)$ for all $z \in S$ if and only if there exists $g \in A(\Delta)$ (resp. $\left.H^{\infty}(\Delta)\right)$ with $g(z)=\zeta(z)$, all $z \in S$.

Indeed, if $g(z)$ is given, then $f(z)=P g(z)=\zeta(z)$ for all $z \in S$.

Corollary 2 strengthens Stout's theorem [7] that if $S$ is a $G$-invariant interpolating set for $H^{\infty}(\Delta)$ it is also an interpolating set for $H^{\infty}(G)$,

Let $a(G) \subset H^{\infty}(G)$ be the subspace of functions continuous in $\bar{R}$, and $a_{0}(G) \subset Q(G)$ the subset of functions analytic in $\bar{R}$.

Corollary 3. $a_{0}(G)$ is dense in $a(G)$.

Proof. We use the notations of $\S$. If $f \in Q(G)$, set $f_{r}(z)=f(r z)$ for $r<1$. Then $P\left(f_{r}\right) \in Q_{0}(G)$. We claim that $P\left(f_{r}\right) \rightarrow P(f)=f$ as $r \rightarrow 1$. For the proof, choose an enumeration $\left\{A_{i}\right\}$ of the elements of $G$, and set $C=\max \left(\|f\|_{\infty}, M\right)$. Given $\epsilon>0$ find $N$ such that

$$
\sup \left\{\sum_{i=N+1}^{\infty}\left|A_{i}^{\prime}(z)\right|^{2}: z \in \Omega\right\}<\epsilon / 4 C \delta\|F\|_{\infty}
$$

and find $r_{0}$ such that for $r_{0}<r<1$

$$
\sup \left\{|f(r z)-f(z)|: z \in \bigcup_{i=1}^{N} A_{i}(\Omega)\right\}<\epsilon / 2 C \delta\|F\|_{\infty} .
$$

Then for $z \in R$ and $r_{0}<r<1$,

$$
\begin{aligned}
\left|P\left(f-f_{r}\right)(z)\right| & \leqq\|F\|_{\infty} \delta \sum_{i=1}^{N}\left|f\left(A_{i} z\right)-f_{r}\left(A_{i} z\right)\right|\left|A_{i}^{\prime}(z)\right|^{2} \\
& +2 C\|F\|_{\infty} \delta \sum_{i=N+1}^{\infty}\left|A_{i}^{\prime}(z)\right|^{2}<\epsilon .
\end{aligned}
$$

For another proof of Corollary 3, see [2, p. 291]. 
5. Remarks. A closer investigation of Forelli's projection of $H^{\infty}(\Delta)$ on to $H^{\infty}(G)$ shows that it too has property (6) but does not extend to $A(\Delta)$. Our projection $P$, like Forelli's projection, has the property that if $f \in L^{p}$ on $\{|z|=1\}, p \geqq 1$ then so is $P f$. In the present case this fact is a simple consequence of the Hölder inequality and the convergence of $\Sigma\left|A^{\prime}(z)\right|$ for $z \in \bar{R}$. In addition, $P$ obviously has the following property. If $A_{p}(\Delta), p \geqq 1$, is the Banach space of analytic functions in $\Delta$ with norm $\iint_{\Delta}|f(z)|^{p} d x d y<\infty$ and $A_{p}(G)$ is the subspace of functions satisfying (3), then $P$ is a bounded projection of each $A_{p}(\Delta)$ on to $A_{p}(G)$.

\section{REFERENCES}

1. N. Alling, A proof of the corona conjecture for finite open Riemann surfaces, Bull. Amer. Math. Soc. 70 (1964), 110-112.

2. - Extensions of meromorphic function rings over non-compact Riemann surfaces. I, Math. Z. 89 (1965), 273-299.

3. L. Bers, Automorphic forms and Poincaré series for infinitely generated Fuchsian groups, Amer. J. Math. 87 (1965), 196-214.

4. C. Earle, A reproducing formula for integrable automorphic forms, Amer. J. Math. 88 (1966), 867-870.

5. O. J. Farrell, On approximation to an analytic function by polynomials, Bull. Amer. Math. Soc. 40 (1934), 908-914.

6. F. Forelli, Bounded holomorphic functions and projections, Illinois J. Math. 10 (1966), 367-380.

7. E. L. Stout, Bounded holomorphic functions on finite Riemann surfaces, Trans. Amer. Math. Soc. 120 (1965), 255-285.

Cornell University and

University of Minnesota 